\title{
Optimisation Studies of Cylinder Water Channel Geometry in an Internal Combustion Engine
}

\author{
Michat Biały ${ }^{1, *}$, and Konrad Pietrykowski ${ }^{1}$ \\ ${ }^{1}$ Lublin University of Technology, Faculty of Mechanical Engineering, Department of Thermodynamics, Fluid Mechanics and \\ Aviation Propulsion Systems, Nadbystrzycka Street 36, 20-618 Lublin, Poland
}

\begin{abstract}
This paper presents an analysis of the results of simulation studies of optimisation of water channel geometry placed in the head of an opposed-piston three-cylinder engine. For this purpose, six computation cases were prepared, differing in shape and cross-section field of channels located along the axis of one of the cylinders. The research was conducted by means of Ansys Flutent software. The conditions of initiation and boundary have been obtained from previous studies using AVL Boost RT software. A fixed temperature was assumed on the cylinder wall and a fixed temperature was assumed for the coolant at the inlet to the model. The studies were carried out for the model of the designed engine, which carries out a two-stroke diesel cycle. The engine will be equipped with direct injection of diesel fuel. On the basis of the conducted analysis of the test results, the flow velocity distributions and distributions of speed fields of the working medium in the critical cross-sections, the distribution of temperature and heat flow fields were prepared. Pressure drops and heat dissipation were also determined for individual models. This provided the basis for the determination of the influence of the rib's transverse geometry shape on the amount of heat received from the cylinder's smoothing walls.
\end{abstract}

\section{Introduction}

Nowadays, experimental research belongs to the group of time- and cost- intensive research. Numerical tests, on the other hand, enable validation of project assumptions at the design stage and thus the verification of the proper functioning of the object. As a result the number of prototypes is reduced as are the costs.

Among the numerical tests we can distinguish computational CFD mechanics, which is currently widely implemented in all areas of life. CFD methods reduce component manufacturing costs. The technology enables smooth modification of the structure, changes in boundary conditions and observation of their effect on key parameters in the analysis of both fluid and energy flows [1].

CFD is employed in numerical testing in mechanical engineering, such as in the analysis of a combustion engine, which generates pressure energy and heat during the burning process in each operating cycle. The obtained gas pressure energy is converted into mechanical energy. The heat, on the other hand, must be dissipated outside the system. More examples of CFD applications are presented in papers [2-4]. In the case of gas flow analysis, the PIV method is a good alternative to CFD, as it allows flow visualisation. The measurement system in question is described in detail in $[5,6]$.

Therefore, it was resolved that simulation tests should be carried out by means of computer fluid mechanics of the quantity of heat flowing from the combustion chamber to the cooling system through a water jacket placed in the engine head. The research object of the study was a newly developed three-cylinder engine with opposite piston arrangement, operating in the diesel circuit [7-10].

\section{CFD analysis}

Model tests of CFD computer fluid mechanics were carried out on the cooling system of a predesigned engine with counter-slip pistons (Fig. 1). The drive unit will be used to drive light windmills. The engine will generate approx. $100 \mathrm{~kW}$ of mechanical power from a capacity of approx. $1600 \mathrm{~cm}^{3}$ and will run a two-stroke diesel cycle with a direct injection of diesel fuel into the combustion chamber. The unit will have three opposed-piston cylinders in a flat arrangement, with two crankshafts opposite each other. This engine may be dedicated for small aircraft including gyroplanes [3]. The use of the piston engine in comparison to turbine engines is basically justified by a longer flight time. This means that during short flights of small regular or unmanned aircrafts and rotorcrafts it is more convenient to apply turbine engines. Unfortunately, the discrepancy in mass decreases with an increase in time of operation. This involves larger unit fuel consumption which is $0.4-0.7 \mathrm{~kg} / \mathrm{kWh}$ and $0.35 \mathrm{~kg} / \mathrm{kWh}$ for the turbine engine and piston engine, respectively $[11,12]$.

The paper analyses the coolant flushing through a section of water jacket for one of the cylinders (Fig. 2). The main aim of the study was to determine the optimal shape and number of water channels distributed along the axis of the cylinder.

\footnotetext{
*Corresponding author: e-mail: m.bialy@pollub.pl
} 


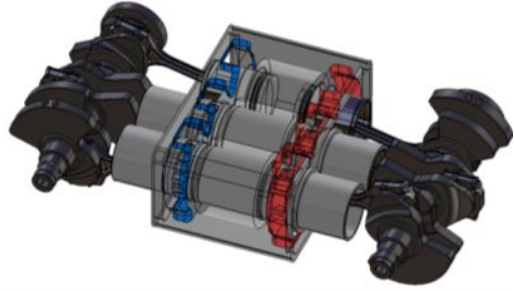

Fig. 1. CAD model of the engine

Simulation tests were carried out with the use of ANSYS software. The research consisted of the following stages [13, 14]:

- preparation of geometry in CAD environment. (The article does not analyse the full model of the water jacket but a section consisting of a single cylinder (Fig. 3)).

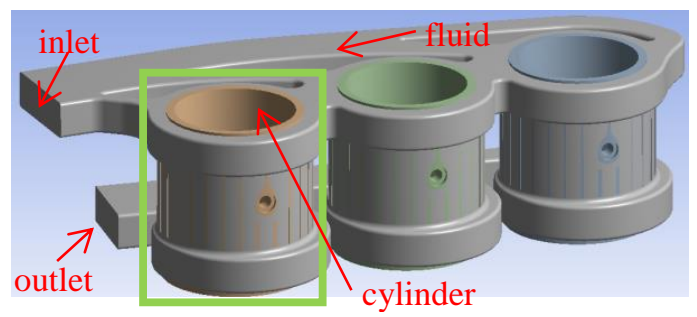

Fig. 2. Full CAD Model of fluid jacket (the frame shows the part under analysis).

- importing geometry into the Geometry module (Fig. 3),

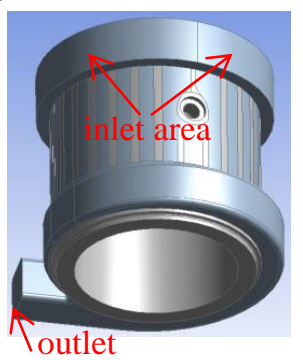

Fig. 3. CAD Model of fluid jacket for example geometry.

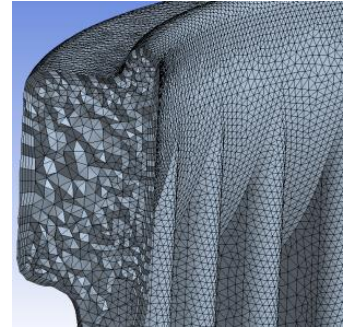

Fig. 4. Cross-section of computational grid of fluid jacket model.
- generation of a grid in the Mesh module (Fig. 4) (tetragonal grid with a five-layer wall layer, growth rate 1.1 , minimum size $0.19 \mathrm{~mm}$ - A more detailed description of the grid can be found in subsequent sections of this paper.)

- specification of initial and boundary conditions: mass flow rate of liquid at the level of $1 \mathrm{~kg} / \mathrm{s}$, medium inlet temperature $90^{\circ} \mathrm{C}$, cylinder wall temperature $480 \mathrm{~K}$.

- conducting simulation of heat flow: assumed heat transfer from steel cylinder (density $7100 \mathrm{~kg} / \mathrm{m}^{3}$, specific heat $460 \mathrm{~J} / \mathrm{kgK}$ ) to medium: water (density $998.2 \mathrm{~kg} / \mathrm{m}^{3}$, specific heat $4182 \mathrm{~J} / \mathrm{kgK}$ ).

- development and analysis of results.

\subsection{Computational model}

In order to verify the effect of the number of ribs and the shape of a single rib geometry (longitudinal channel), six design cases were subjected to simulation tests:

- 18 ribs per cylinder (Fig. 5),

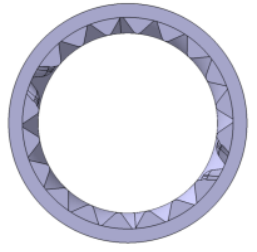

Fig. 5. The cross-section of the cylinder: 18 ribs per cylinder.

- 22 ribs per cylinder (Fig. 6),

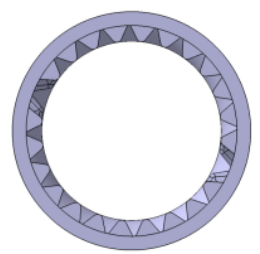

Fig. 6. The cross-section of the cylinder: 22 ribs per cylinder.

- 26 ribs per cylinder, 26 ribs $-1 \mathrm{~mm}$ - the distance between the rib bases $-1 \mathrm{~mm}$ (Fig. 7),

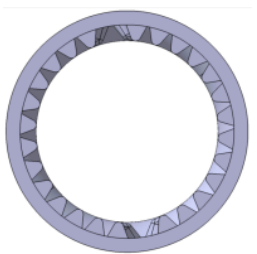

Fig. 7. The cross-section of the cylinder: 26 ribs per cylinder $1 \mathrm{~mm}$.

- 26 ribs $-2.5 \mathrm{~mm}$ - the distance between the rib bases $-2.5 \mathrm{~mm}$ (Fig. 8),

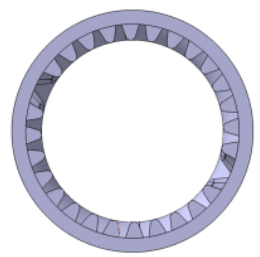

Fig. 8. The cross-section of the cylinder: 26 ribs per cylinder $2.5 \mathrm{~mm}$.

- 26 ribs $-3 \mathrm{~mm}$ - the distance between the rib bases $3 \mathrm{~mm}$ (Fig. 9),

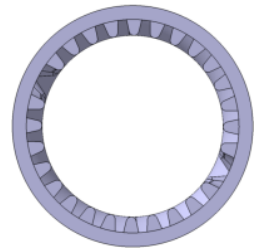

Fig. 9. The cross-section of the cylinder: 26 ribs per cylinder $3 \mathrm{~mm}$.

- 30 ribs per cylinder (Fig. 10),

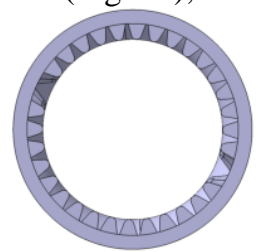

Fig. 10. The cross-section of the cylinder: 30 ribs per cylinder 


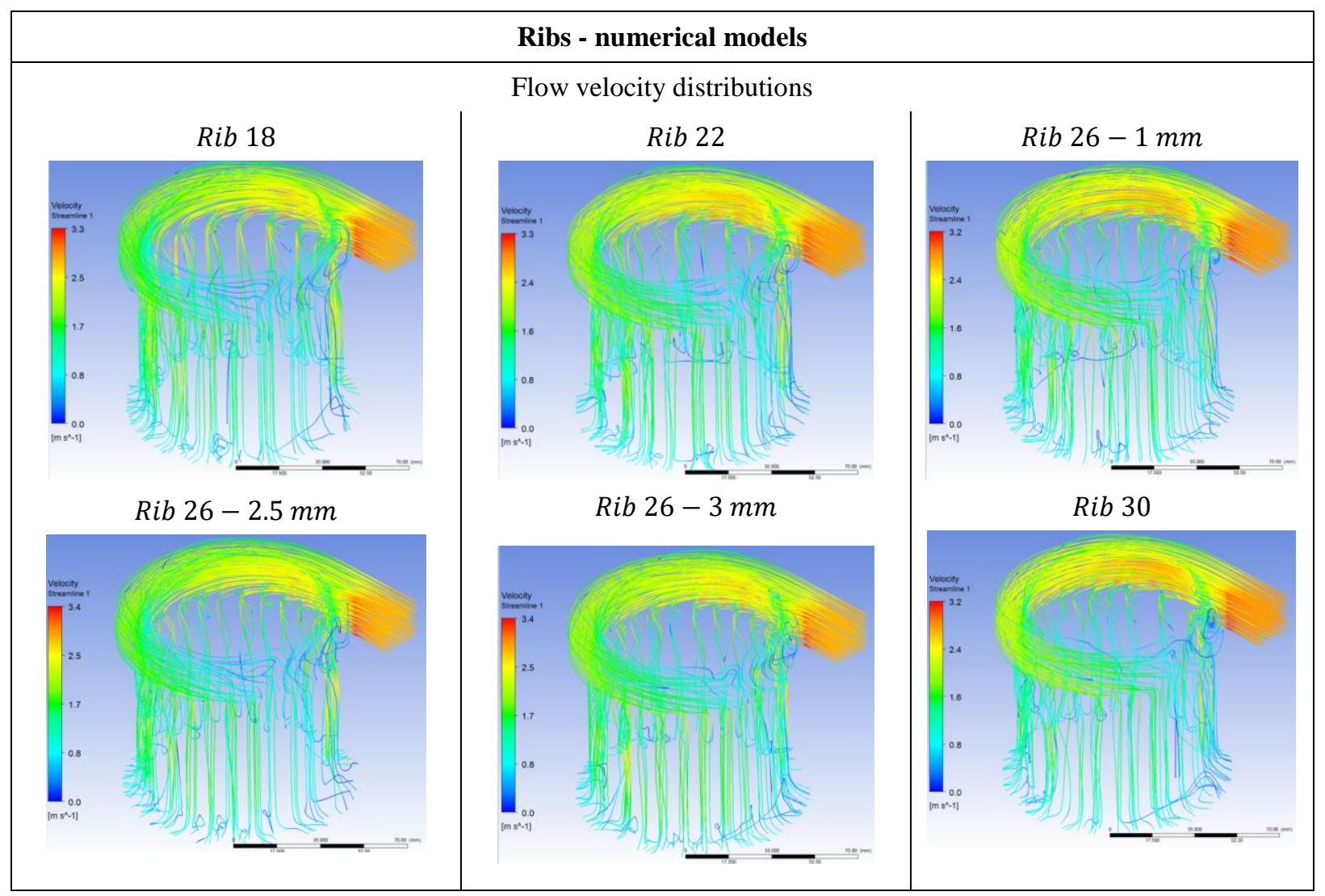

Fig. 11. Velocity distributions for computational models of ribs.

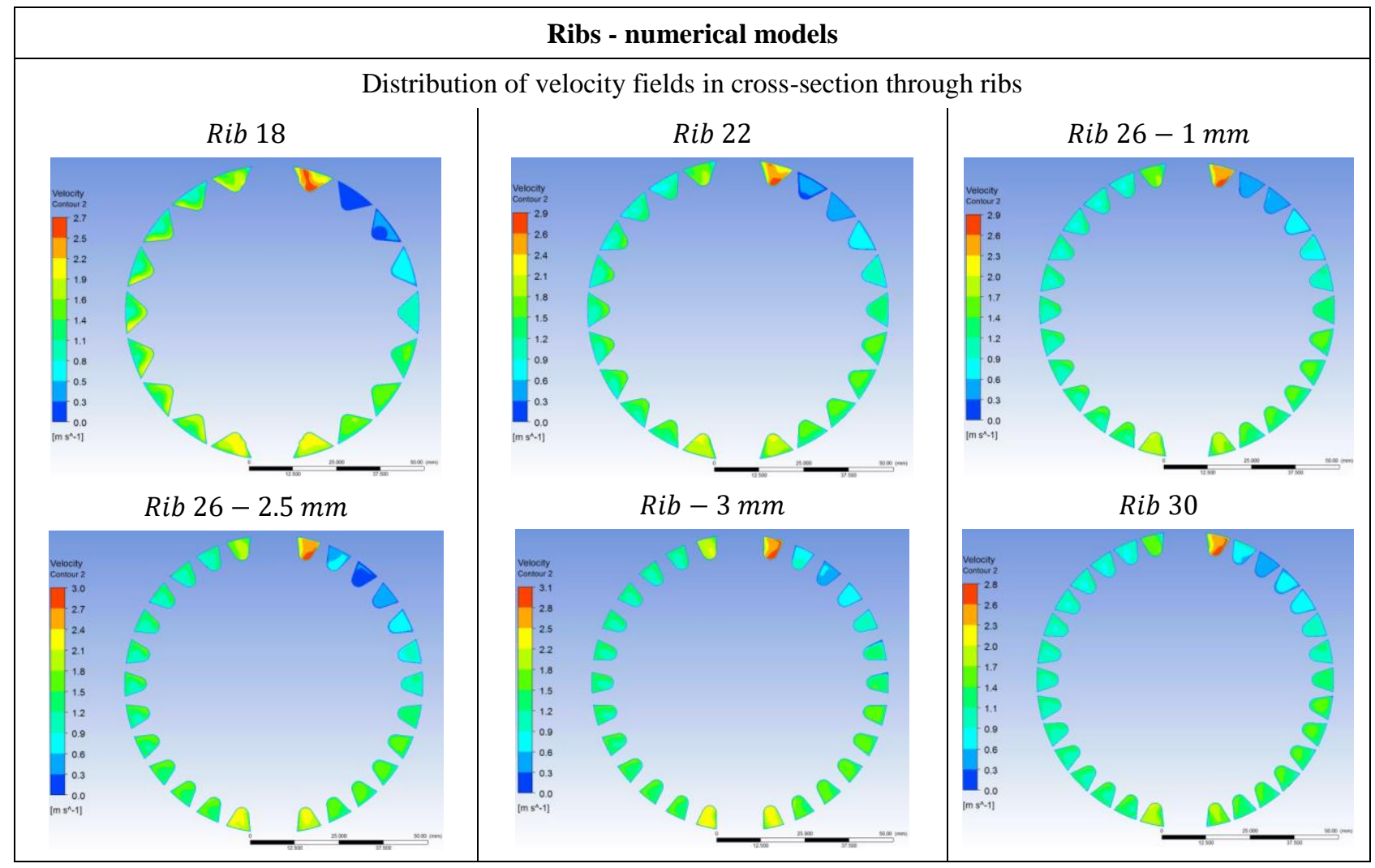

Fig. 12. Distribution of velocity fields in cross-section through ribs for the rib computational models. 


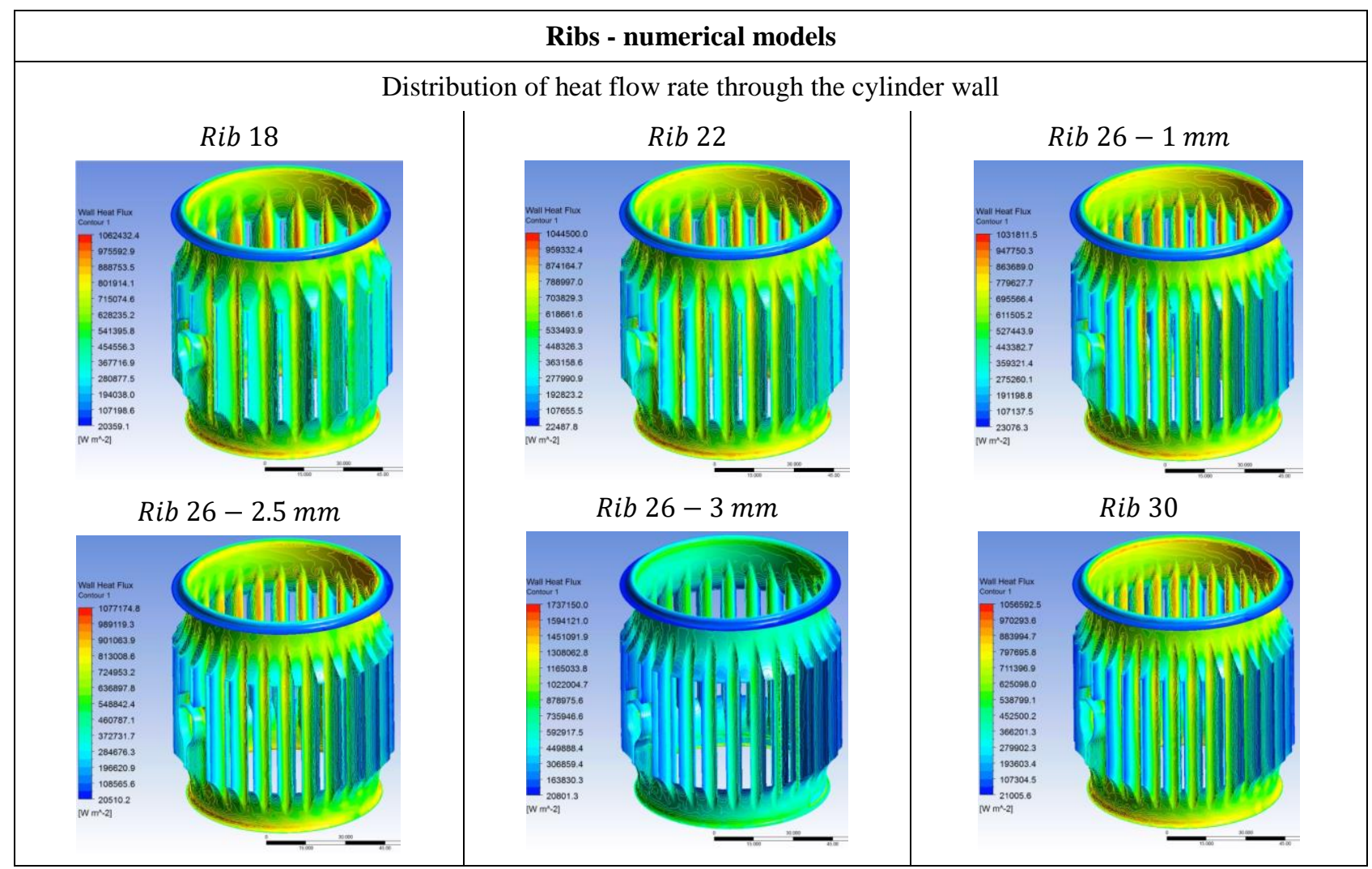

Fig. 13. Distribution of heat flow rate through the cylinder wall for the rib computational models.

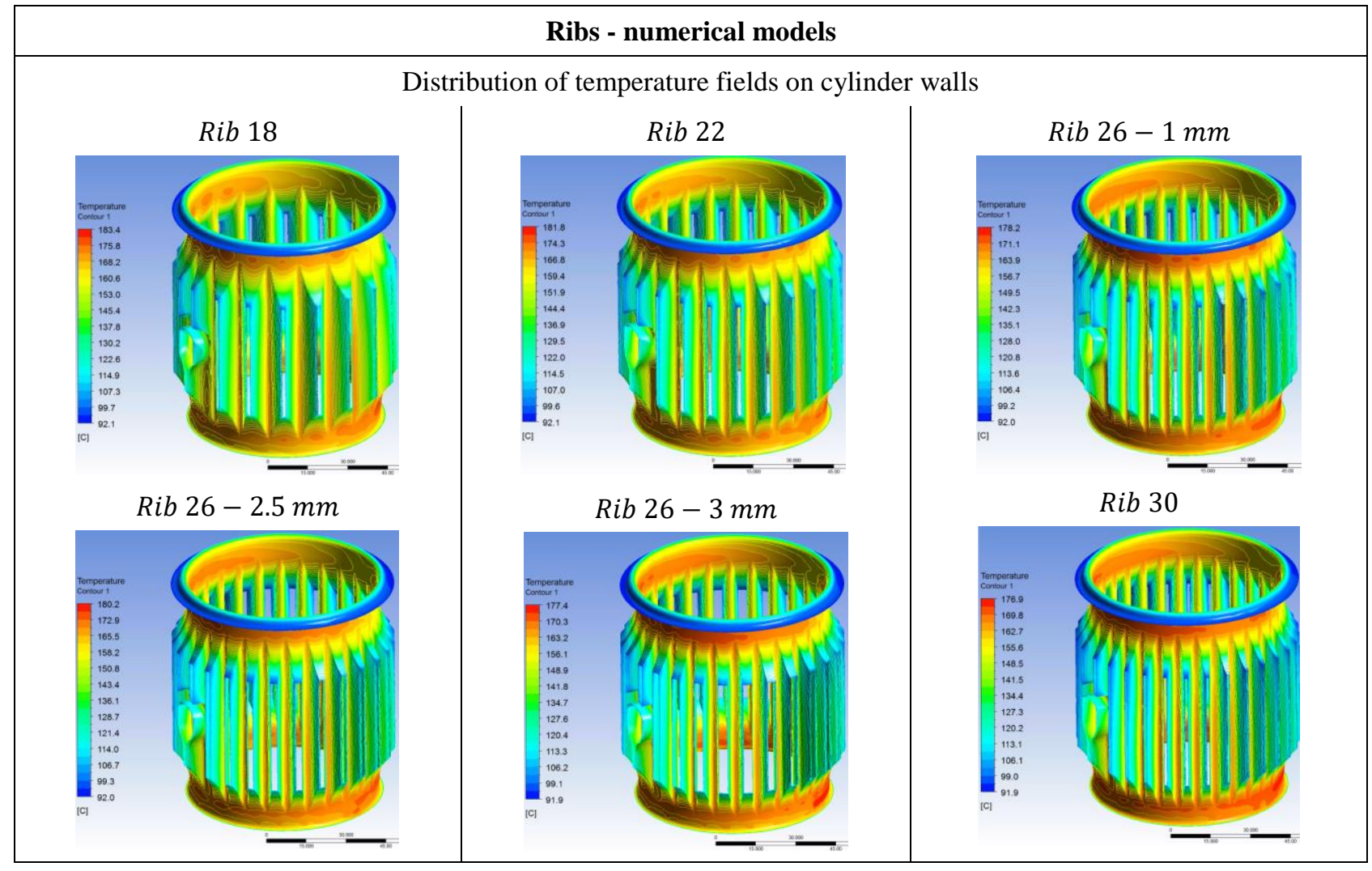

Fig. 14. Distribution of temperature fields on cylinder walls for the rib computational models. 


\subsection{Test procedures}

The simulations were conducted with the use of ANSYS FLUENT software. The following assumptions were made:

- cylinder wall temperature: $207^{\circ} \mathrm{C}$ (average cycle temperature),

- inlet temperature of the working medium: $90^{\circ} \mathrm{C}$,

- coolant mass flow rate: $1 \mathrm{~kg} / \mathrm{s}$,

- intensity of turbulence: $5 \%$,

- viscosity index of turbulence: $10 \%$,

- outlet temperature $95^{\circ} \mathrm{C}$ (Result parameter).

The assumptions above have been derived from the previously conducted research on the engine designed with the application of AVL Boost RT software [15].

This tool is a fully integrated simulation software for internal combustion engines. It includes advanced models that provide accurate prediction of engine performance, exhaust emissions and acoustics. [16]

For simulation studies, averaged shoot equations and the model of turbulence of k-epsilon were used [17]. A real k-epsilon model with standard wall functions was included. The studies were conducted to obtain a maximum of 3,000 computational steps of the residuals convergence. The research was carried out for walls with adiabatic features. The grid consisted of 130,000 to 140,000 nodes and 5.4 million to 6 million elements.

\subsection{Test results}

The conducted numerical simulations produced the following data:

- flow velocity distributions (Fig. 11),

- the distribution of the speed fields in cross-section through the central part of the rib (Fig. 12),

- distributions of the heat flow through the cylinder wall (Fig. 13),

- distributions of temperature fields on the cylinder walls (Fig. 14).

\section{Analysis of results and summary}

The effect of the transverse rib shape and its quantity on the ability to receive heat from hot cylinder walls were determined from the simulation studies. For this purpose, the test results for six different geometries were analysed. These structures differed in the number of ribs and in their cross-sectional area.

For all cases (Table 1) the pressure drop between inlet and outlet does not exceed $278 \mathrm{~Pa}$. The maximum temperature drop spread was below $0.1^{\circ} \mathrm{C}$ for the same starting and boundary conditions. From the boiler, the difference between the maximum and minimum heat output was $1.29 \mathrm{~kW}$. This difference constitutes slightly more than $8 \%$ of the difference between the different fin versions for a single cylinder. In terms of engine power $(100 \mathrm{~kW})$, this represents less than $1.3 \%$ of the mechanical power generated by the drive unit. Therefore, it was considered that the shape of the transverse geometry of the ribs did not affect the amount of heat absorbed from the cylinder's smoothing walls.
Table 1. Comparison of parameters characteristic for the analysed computation cases.

\begin{tabular}{|c|c|c|c|}
\hline \multirow{2}{*}{$\begin{array}{c}\text { Computation } \\
\text { case }\end{array}$} & $\begin{array}{c}\Delta \mathbf{p} \\
{[\mathbf{k P a}]}\end{array}$ & $\Delta \mathbf{T}\left[{ }^{\mathbf{0}} \mathbf{C}\right]$ & Pthermal $\left.^{\mathbf{k} W}\right]$ \\
\cline { 2 - 4 } & \multicolumn{3}{|c|}{$\mathbf{m}=\mathbf{1} \mathbf{~ k g} / \mathbf{s}$} \\
\hline $\mathbf{1 8}$ ribs & 0.795 & 4.639 & 14.33 \\
\hline $\mathbf{2 2}$ ribs & 1.007 & 4.621 & 14.79 \\
\hline $\begin{array}{c}\mathbf{2 2} \text { ribs } \\
(\mathbf{1 . 0} \mathbf{~ m m})\end{array}$ & 1.074 & 4.604 & 15.18 \\
\hline $\begin{array}{c}\mathbf{2 2} \text { ribs } \\
(\mathbf{2 . 5} \mathbf{~ m m})\end{array}$ & 1.109 & 4.689 & 15.22 \\
\hline $\begin{array}{c}\mathbf{2 2} \text { ribs } \\
(\mathbf{3 . 0} \mathbf{~ m m})\end{array}$ & 1.619 & 4.630 & 15.62 \\
\hline $\mathbf{3 0}$ ribs & 1.073 & 4.640 & 15.54 \\
\hline
\end{tabular}

This work has been realised in the cooperation with The Construction Office of WSK "PZL-KALISZ" S.A." and is part of Grant Agreement No. POIR.01.02.00-00-0002/15 financed by the Polish National Centre for Research and Development.

\section{References}

1. A.B. Chougule, R. Suresh, C.A. Vinay, Proceedings of the $43^{\text {rd }}$ National conference on fluid mechanics and fluid power. December 15-17, 2016, Mnnita, Allahabad, FMFP2016, 35 (2016)

2. Ł. Grabowski, Z. Czyż, K. Kruszczyński, SAE 2014 International Powertrain, Fuels \& Lubricants Meeting, Birmingham, United Kingdom (2014)

3. Z. Czyż, P. Magryta, M. Szlachetka. Adv. Sci. Tech. Research Journal 9 (26), 89-95 (2015) DOI: $10.12913 / 22998624 / 2370$

4. Z. Czyż, P. Karpiński, T. Łusiak, T. Szczepanik. ITM Web of Conferences 15, 07008 (2017) W. Stryczniewicz, K. Surmacz, Prace Instytutu Lotnictwa 235, 17-27 (2014)

5. Z. Czyż, W. Stryczniewicz, Adv. Sci. Tech. Research Journal 12 (1), 106-114 (2018)

6. A. Alqahtani, F. Shokrollahihassanbarough, M.L. Wyszynski, Combustion Engines, 161 (2), 68-72 (2015)

7. M. Biały, K. Pietrykowski, T. Tulwin, P. Magryta, Combustion Engines, 170 (3), 8-18 (2017)

8. Z. Czyż, T. Łusiak, D. Czyż, D. Adv. Sci. Tech. Research Journal 10 (31), pages 169-176, (2016)

9. K. Pietrykowski, T. Tulwin. SAE Int. J. Engines. 1 (8), 82-88 (2015)

10. Z. Czyż, P. Magryta, Eksploat. Niezawodn. 18 (4): 507-513 (2016)

11. M. Wendeker, Z. Czyż, Eksploat. Niezawodn., 18 (1), 89-97 (2016).

12. www.ansys.com.

13. R.S. Nursal, A.H. Hashim, N.I. Nordin, M. Hamid, M.R. Danuri. ARPN IJEAS, 12 (4) (2017)

14. Ł. Grabowski, K. Pietrykowski, P. Karpiński. ITM Web Of Conferences (15), 2017.

15. www.avl.com/boost.

16. V.A. Romanov, N.A. Khozeniuk. ICIE, Procedia Eng. 150, 490 - 496 (2016). 